\title{
Shilnikov chaos, Filippov sliding and boundary equilibrium bifurcations
}

\author{
P. A. GLENDINNING \\ School of Mathematics, University of Manchester, Manchester M13 9PL, UK \\ email:p.a.glendinning@manchester.ac.uk
}

(Received 28 September 2017; revised 11 May 2018; accepted 15 May 2018; first published online 13 June 2018)

\begin{abstract}
In the 1960s, L.P. Shilnikov showed that certain homoclinic orbits for smooth families of differential equations imply the existence of chaos, and there are complicated sequences of bifurcations near the parameter value at which the homoclinic orbit exists. We describe how this analysis is modified if the differential equations are piecewise smooth and the homoclinic orbit has a sliding segment. Moreover, we show that the Shilnikov mechanism appears naturally in the unfolding of boundary equilibrium bifurcations in $\mathbb{R}^{3}$.
\end{abstract}

Key words: Bifurcation theory, homoclinic orbits, piecewise smooth dynamics.

\section{Introduction}

This paper brings together three important themes of smooth and piecewise smooth dynamical systems: Shilnikov homoclinic orbits, Filippov sliding flows and boundary equilibrium bifurcations (BEBs). The results presented below show that complicated (chaotic) motion can exist as a result of BEBs in three-dimensional systems.

The dynamics of piecewise smooth differential equations is defined by different smooth vector fields in different open regions of phase space separated by switching surfaces. Although they are used to model many mechanical, biological and control systems, the bifurcation theory associated with such systems is still being developed. Filippov [12] provides a very thorough classification of planar systems, but in higher dimensions relatively little is known, and it may be that there is not enough regularity to avoid a zoological description rather than the relatively concise description of bifurcations for typical families of smooth systems. Even in the planar case, if a stationary point of one equation intersects the switching surface (the boundary between regions in which different differential equations govern the flow), there are 12 different types of codimension one bifurcation $[12,15,18]$. These are called planar BEBs. One of the aims of this paper is to describe bifurcation mechanisms associated with BEBs in $\mathbb{R}^{3}$.

A feature of piecewise smooth differential equations is that there may be some parts of the switching surface on which the normal components to the switching surface of the vector fields defining the differential equations on each side of the switching surface are anti-parallel. If both point towards the manifold, then solutions typically hit the switching surface in finite time and then some new rule needs to be introduced if the solutions are to be continued. Filippov [12] described a natural evolution on the switching manifold 
in such cases by choosing a linear combination of the two vector fields such that the component normal to the switching surface is zero. This defines the Filippov sliding flow on the switching surface, although it is possible to adopt other conventions.

The analysis of homoclinic bifurcations of smooth systems in dimension greater than two was pioneered by L.P. Shilnikov in the 1960s. These are examples of global bifurcations in which there is a special parameter value at which a solution (a homoclinic orbit) is asymptotic to a stationary point of the flow in forwards and backwards time. For typical families of smooth differential equations (e.g. non-Hamiltonian systems), this is a codimension one phenomenon and Shilnikov [29,30] showed that in a neighbourhood of the parameter for which a homoclinic orbit exists then, under conditions described in Section 2.1, there are chaotic solutions. Glendinning and Sparrow [16] (see also [13]) showed that there are infinite sequences of saddle node bifurcations on either side of the bifurcation value as a periodic orbit winds its way to infinite period. Moreover, there are infinite sequences of parameters accumulating on the homoclinic bifurcation value at which the system has more geometrically complicated homoclinic bifurcations. These loop several times through a neighbourhood of the stationary point and are called $n$-pulse homoclinic orbits, where $n$ is the number of loops made.

It is natural to ask how the analysis of Shilnikov's chaotic case is changed if the system is piecewise smooth. If there is no sliding locally, then piecewise linear models have already been used extensively in the study of homoclinic bifurcations $[1,2,14,23]$ and more detailed statements in the context of three-dimensional piecewise smooth systems with no sliding have also been made [22]. Hös and Champneys [19] conjecture that a Shilnikov type orbit exists in an impact model, whilst Novaes and Teixeira [24] describe Shilnikov bifurcations to pseudostationary points in the sliding region.

If the homoclinic orbit has a sliding segment, this effectively reduces the dimension of the flow by one and it might be thought that the introduction of such a segment could not generate interesting dynamics for flows in $\mathbb{R}^{3}$. However, different trajectories of the three-dimensional part of the flow may map to the same sliding trajectory, and it turns out that the return map on an appropriate part of phase space is essentially an approximate one-dimensional map that arises in Shilnikov's analysis (more accurately, it arises as a fixed point equation - see [16] and Section 6). This implies the existence of infinitely many saddle node bifurcations locally, and infinitely many bifurcations involving more complicated sliding homoclinic orbits as in the smooth case.

The new results are stated in Section 2 along with a brief account of the standard theory of Shilnikov and Filippov. In Section 3, we show numerically that sliding homoclinic orbits occur in the unfolding of a three-dimensional BEB, and hence that the analysis is central to other bifurcations of piecewise smooth systems. Numerical confirmation of the results are given in Sections 4-6 provide the theoretical underpinning.

\section{Background and statement of results}

The description of the results of this paper requires two ingredients: the classic results of Shilnikov on homoclinic chaos and the equally classic approach of Filippov to sliding in piecewise smooth systems. In the first two subsections of this section, the essential 
ingredients of these areas are described and then they are brought together in the final subsection which contains the new results of this paper.

\subsection{Shilnikov chaos}

A stationary point of a differential equation in $\mathbb{R}^{3}$ is a saddle-focus if the Jacobian matrix of the flow evaluated at the stationary point has eigenvalues $\lambda$ and $-\rho \pm i \omega$ with $\lambda, \rho, \omega>0$ by a choice of the direction of time. A solution $\Gamma(t)$ is a homoclinic orbit if there is a stationary point $\mathbf{x}^{*}$ such that $\lim _{t \rightarrow \pm \infty} \Gamma(t)=\mathbf{x}^{*}$ and $\Gamma(0) \neq \mathbf{x}^{*}$. A neighbourhood $\Gamma_{\epsilon}$ of the homoclinic orbit $\Gamma$ is the set

$$
\Gamma_{\epsilon}=\left\{x\left|\min _{t \in \mathbb{R}}\right| x-\Gamma(t) \mid<\epsilon\right\}
$$

A chaotic set of solutions is an invariant set which contains a subset on which the dynamics of an appropriately chosen return map is equivalent to a full shift on two symbols.

Different degrees of smoothness are used in the statement of results by different authors, from analytic [13] to $C^{1,1}[32,33]$. The choice made here is $C^{3}$, and this is justified in the technical remark of Section 5 .

Theorem 2.1 ([29,30]) Consider a family of $C^{3}$ vector fields $f: \mathbb{R}^{3} \times \mathbb{R} \rightarrow \mathbb{R}^{3}$ depending on a real parameter $\mu$ such that the origin $\mathbf{0} \in \mathbb{R}^{3}$ is a saddle-focus for $|\mu|$ sufficiently small and the eigenvalues of the Jacobian at $\mu=0$ are $\lambda,-\rho \pm i \omega$ with $\lambda>\rho>0$. Suppose that if $\mu=0$, then the differential equation $\dot{\mathbf{x}}=f(\mathbf{x}, \mu)$ has a homoclinic orbit to the stationary point at the origin and there are no homoclinic orbits passing once close to $\Gamma(t)$ for $|\mu| \neq 0$ sufficiently small. Then there exists $\epsilon>0$ such that if $|\mu|$ is sufficiently small, an unstable set of chaotic solutions exists in $\Gamma_{\epsilon}$.

Much more can be said about the nature of the chaotic set, see [2,30] for details. Subsequent work described some of the bifurcations in $\Gamma_{\epsilon}$.

Theorem 2.2 ( $[13,16])$ Consider a family of $C^{3}$ vector fields $f: \mathbb{R}^{3} \times \mathbb{R} \rightarrow \mathbb{R}^{3}$ satisfying the conditions of Theorem 2.1. Then there is a family of periodic orbits which pass once through $\Gamma_{\epsilon}$ in each period and which are continuously connected as a function of $\mu$ and a set of parameter values $\mu_{n} \rightarrow 0$ as $n \rightarrow \infty, \mu_{2 n+1}<0<\mu_{2 n}$ such that the family has a saddle node bifurcation at $\mu_{n}$ and

$$
\lim _{n \rightarrow \infty} \frac{\mu_{n}-\mu_{n+1}}{\mu_{n-1}-\mu_{n}}=\exp \left(-\frac{\pi \rho}{\omega}\right)
$$

Also, there is a sequence $\tilde{\mu}_{n}$ tending to zero from one side such that if $\mu=\tilde{\mu}_{n}$, then the differential equation has a homoclinic orbit that passes twice through $\Gamma_{\epsilon}$, with

$$
\lim _{n \rightarrow \infty} \frac{\tilde{\mu}_{n}-\tilde{\mu}_{n+1}}{\tilde{\mu}_{n-1}-\tilde{\mu}_{n}}=\exp \left(-\frac{2 \pi \lambda}{\omega}\right) .
$$


The curve of periodic orbits described in this theorem can be represented conveniently in parameter $(\mu)$ period $(T)$ space where it is approximately

$$
\mu \sim A e^{-\rho T} \cos (\omega T+\Phi)
$$

for constants $A$ and $\Phi$ when $T$ is large enough. As $T \rightarrow \infty, \mu \rightarrow 0$ and so the orbit approaches the homoclinic orbit which can be thought of intuitively as a periodic orbit of infinite period. The homoclinic orbits that pass twice through $\Gamma_{\epsilon}$ are called double-pulse homoclinic orbits; the sign of $\mu$ for which they exist is determined by the geometry of the unstable manifold, see e.g. [16].

\subsection{Filippov flows}

Let $F^{ \pm}: \mathbb{R}^{3} \times \mathbb{R} \rightarrow \mathbb{R}^{3}$ be smooth vector fields parameterized by a real parameter $v$ and let $s: \mathbb{R}^{3} \times \mathbb{R} \rightarrow \mathbb{R}$ be smooth. The introduction of a different symbol ( $v$ rather than $\mu$ ) to represent the parameter is so that there is no confusion with the role of the parameter $\mu$ for the sliding Shilnikov orbits of the previous sections. Bifurcation theory for piecewise smooth systems describes the changes of the dynamics of systems defined by

$$
\dot{\mathbf{x}}=\left\{\begin{array}{l}
F^{+}(\mathbf{x}, v), \text { if } s(\mathbf{x}, v)>0 \\
F^{-}(\mathbf{x}, v), \text { if } s(\mathbf{x}, v)<0
\end{array}\right.
$$

as the parameter $v$ is varied. The surface $s(\mathbf{x}, v)=0$ is called the switching surface. Solutions in the regions defined by $s(\mathbf{x}, v)<0$ and $s(\mathbf{x}, v)>0$ exist and are unique until they intersect the switching surface. Let $\langle.,$.$\rangle denote the standard inner product in \mathbb{R}^{3}$, then a solution at the switching surface with

$$
\left\langle F^{+}, \nabla s\right\rangle\left\langle F^{-}, \nabla s\right\rangle>0
$$

has a component crossing the switching surface in the same direction from each side and so the solution can be continued across the boundary. The only complication for stability calculations is the difference in time at which nearby solutions strike the switching manifold, which means that local return maps are modified by a saltation matrix [6]. Homoclinic bifurcations in systems with only this type of transition include the piecewise linear examples of $[1,2,14]$, and a detailed analysis is given in [22].

If

$$
\left\langle F^{+}, \nabla s\right\rangle\left\langle F^{-}, \nabla s\right\rangle<0,
$$

then stable (if both vector fields point towards the switching surface) or unstable (if both point away from the switching surface) sliding motion can be defined, in which the dynamics is constrained to lie on the switching surface. Filippov [12] defined a natural way to describe the sliding motion and although his choice is by no means unique, it is the standard evolution and will be accepted here without further justification. The idea is to choose a linear combination of the two vector fields, $\alpha F^{+}+(1-\alpha) F^{-}$such that

$$
\left\langle\alpha F^{+}+(1-\alpha) F^{-}, \nabla s\right\rangle=0
$$




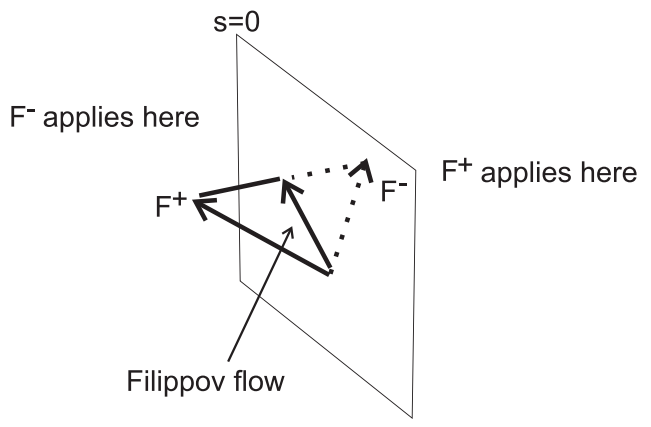

FIGURE 1. Geometry of the Filippov construction creating the Filippov flow satisfying (2.6).

on $s(\mathbf{x}, v)=0$ so that the flow on the switching surface is tangential to the switching surface as shown in Figure 1. In this case, if

$$
\dot{\mathbf{x}}=\alpha F^{+}(\mathbf{x}, v)+(1-\alpha) F^{-}(\mathbf{x}, v)
$$

with $\alpha$ determined by (2.6), then

$$
\frac{d}{d t} s(\mathbf{x}, v)=\left\langle\alpha F^{+}+(1-\alpha) F^{-}, \nabla s\right\rangle=0 .
$$

Note that (2.6) always has a solution $\alpha \in(0,1)$ if $(2.5)$ holds. The evolution defined by (2.7) is called the sliding or Filippov flow on the switching manifold. A stable sliding motion typically terminates at a locus of either

$$
\left\langle F^{+}, \nabla s\right\rangle=0 \quad \text { or } \quad\left\langle F^{-}, \nabla s\right\rangle=0
$$

on $s(\mathbf{x}, v)=0$, where one of the vector fields is tangential to the switching surface. These are called the tangency sets or fold loci and more complicated situations such as the intersection of these sets are in the process of being understood [5,20] though this will not be an issue below.

A stationary point of (2.7) which is not a stationary point of the full system (2.3) is called a pseudostationary point of the Filippov flow.

\subsection{Sliding homoclinic orbits}

The first new result of this paper concerns the consequences of the existence of a homoclinic orbit of Shilnikov type which has a sliding segment. This means that locally solutions are restricted to a two-dimensional surface and hence the two-dimensional return map of the smooth case is replaced by a one-dimensional return map. It is not immediately obvious whether this makes important changes to the implications of the existence of such orbits. We show below that it does not.

Suppose that if $\mu=0$, the differential equation (2.3) defined by smooth functions $F^{ \pm}$ and switching surface $s$ has a stationary point $\mathbf{x}^{*}$ with $s\left(\mathbf{x}^{*}, 0\right)>0$. Then there is a sliding homoclinic orbit to $\mathbf{x}^{*}$ if there is a solution (in the sense of the previous section) $\Gamma(t)$, and $t_{1}<t_{2}$, such that 
(i) if $-\infty<t<t_{1}$, then $s(\Gamma(t), 0)>0$, moreover, $\lim _{t \uparrow t_{1}} s(\Gamma(t), 0)=0$ and $\lim _{t \rightarrow-\infty} \Gamma(t)=\mathbf{x}^{*}$

(ii) if $t_{2}<t<\infty$, then $s(\Gamma(t), 0)>0$, moreover, $\lim _{t \downarrow t_{2}} s(\Gamma(t), 0)=0$ and $\lim _{t \rightarrow \infty} \Gamma(t)=$ $\mathbf{x}^{*}$;

(iii) if $t_{1}<t<t_{2}$, then $\Gamma(t)$ lies in a stable sliding region of $s(\mathbf{x}, 0)=0$ and satisfies (2.7);

(iv) $\Gamma\left(t_{1}\right)$ does not lie on a fold locus;

(v) $\Gamma(t)$ is bounded away from any pseudostationary points of the flow.

Note that conditions (ii) and (iii) imply that the fold locus at $\Gamma\left(t_{2}\right)$ is a visible fold for $F^{+}$in the language of [5] and the conditions could be rephrased as a curvature condition in terms of the derivatives of $F^{+}$. These conditions describe the simplest case, but orbits with extra non-sliding intersections with the switching surface could be analysed analogously. With this definition, the following result holds for sliding homoclinic orbits.

Theorem 2.3 Suppose that if $\mu=0$, a family of piecewise smooth differential equations (2.3) has a sliding homoclinic orbit $\Gamma(t)$ to a stationary point $\mathbf{x}^{*}$ with $s\left(\mathbf{x}^{*}, 0\right)>0$ which is a saddle-focus with $\lambda>\rho$ and satisfies ( $i)-(v)$ above. Suppose further that there are no homoclinic orbits passing once close to $\Gamma(t)$ if $|\mu|$ is sufficiently small. Then the conclusions of Theorems 2.1 and 2.2 hold.

This shows that despite the collapse to two dimensions the chaotic nature of the saddle-focus is preserved, and the description of the recurrent dynamics is obtained from a one-dimensional map. The proof of Theorem 2.3 is deferred to the end of this paper, Sections 5 and 6 , so that we can emphasize that sliding homoclinic bifurcations exist in examples relevant to BEBs.

\subsection{Boundary equilibrium bifurcations}

A BEB occurs in piecewise smooth systems of the form (2.3) with parameter $v$ if there exists a parameter value, $v=0$ say, and $\mathbf{x}^{*} \in \mathbb{R}^{3}$ such that $F^{+}\left(\mathbf{x}^{*}, 0\right)=\mathbf{0}$ and $s\left(\mathbf{x}^{*}, 0\right)=0$, and a transversality condition holds, so that if $v<0$, then there are no stationary points of $F^{+}$in $s(\mathbf{x}, v)>0$ near $\mathbf{x}^{*}$, and if $v>0$ there is one such stationary point locally. In Section 3, we show numerically that there exist BEBs which have a sliding homoclinic orbit of the type described in previous sections (Theorem 2.3), thus BEBs provide a natural source of sliding homoclinic orbits.

Conjecture. There exists an open set of BEBs in three-dimensional Filippov systems such that on one side of the bifurcation the Filippov flow has a parameter at which a sliding homoclinic orbit to a saddle-focus exists and satisfies the Shilnikov condition $\rho<\lambda$.

We will give very strong numerical evidence the existence of such BEBs in Section 3. This depends on showing that there exists a parameter at which a solution of a twodimensional linear differential equation connecting two points that are known analytically. This is such a simple shooting problem (looking for a parameter at which a solution passes on one side of the target point and another at which it passes on the other side of the point in a one-parameter family) that we consider the existence of BEBs in the conjecture 
to be effectively demonstrated. An example of an approximate sliding homoclinic orbit is shown in Figure 2.

The idea that these BEBs form an open set in the space of BEBs will be supported by considering the effect of perturbations on the argument used to construct the sliding homoclinic connections in the approximate equations of Section 3. This argument is made at the end of Section 3 .

\section{Boundary equilibrium bifurcations with sliding homoclinic orbits}

In this section, a simple example is given of a BEB with a (numerically verified) sliding homoclinic orbit satisfying the conditions of Shilnikov's Theorem.

Consider the Filippov system defined by

$$
F^{+}(\mathbf{x}, v)=\left(\begin{array}{ccc}
-\rho & a & -\omega \\
0 & \lambda & 0 \\
\omega & b & -\rho
\end{array}\right)\left(\begin{array}{c}
x \\
y \\
z-v
\end{array}\right)+O\left((|\mathbf{x}|+|v|)^{2}\right)
$$

where $\rho, \lambda$ and $\omega$ are positive constants and $a$ and $b$ are arbitrary constants, and

$$
F^{-}(\mathbf{x}, v)=\left(\begin{array}{c}
U_{1} \\
U_{2} \\
U_{3}
\end{array}\right)+O((|\mathbf{x}|+|v|))
$$

and with switching surface defined by the function

$$
s(\mathbf{x}, v)=z .
$$

The constants $\rho, \lambda, \omega$ and $U_{3}$ are positive so that the Jacobian matrix of (3.1) has eigenvalues $\lambda$ and $-\rho \pm i \omega$, and the flow in $z<0$ is directed upwards at $z=0$. The remaining constants $a, b, U_{1}$ and $U_{2}$ are arbitrary, and $v$ is the parameter unfolding the bifurcation. In the Appendix, we show that this is the generic local normal form for the BEB to a saddle-focus.

If $v>0$, there is a stationary point $(0,0, v)$ in $z>0$, whilst if $v<0$, the stationary point of $F^{+}$is in $z<0$ where $F^{-}$applies and so it is not a stationary point of the piecewise smooth system. A BEB occurs at $v=0$.

To determine conditions for the existence of a Shilnikov sliding homoclinic orbit if $v>0$, the geometry of the stable and unstable manifolds of the stationary point and sliding regions on the switching surface $z=0$ need to be described. Let $F^{ \pm}=\left(F_{1}^{ \pm}, F_{2}^{ \pm}, F_{3}^{ \pm}\right)$.

By assumption

$$
U_{3}>0
$$

so the switching surface is attracting from below locally, then sliding occurs on $z=0$ if $F_{3}^{+}(x, y, 0 ; v)<0$, i.e. if

$$
\omega x+b y+\rho v<0
$$

with boundary

$$
W_{b}: \quad \omega x+b y+\rho v=0 .
$$


The eigenvalues of the linear part of (3.1) are $-\rho \pm i \omega$ and $\lambda$ so suppose

$$
0<\rho<\lambda \text {. }
$$

The stable manifold of $(0,0, v)$ is $y=0$ which intersects the switching surface $(z=0)$ on the line

$$
W_{s}: \quad y=0,
$$

and a short calculation shows that the unstable manifold is the line

$$
\left(\begin{array}{l}
0 \\
0 \\
v
\end{array}\right)+r\left(\begin{array}{c}
a(\lambda+\rho)-b \omega \\
(\lambda+\rho)^{2}+\omega^{2} \\
b(\lambda+\rho)+a \omega
\end{array}\right)
$$

which intersects the switching manifold at the point $W_{u}=\left(x_{0}, y_{0}, 0\right)$, where

$$
x_{0}=-v\left(\frac{a(\lambda+\rho)-b \omega}{b(\lambda+\rho)+a \omega}\right), \quad y_{0}=-v\left(\frac{(\lambda+\rho)^{2}+\omega^{2}}{b(\lambda+\rho)+a \omega}\right) .
$$

Thus, a sliding homoclinic orbit exists if $v>0, W_{u}$ satisfies (3.4) and there is a sliding solution in (3.4) from $W_{u}$ to the point on the intersection of $W_{s} \cap W_{b}$, i.e. from $W_{u}$ to $W_{p}=\left(x_{p}, 0,0\right)$, where

$$
x_{p}=-\frac{\rho v}{\omega} .
$$

The sliding flow of (2.7) on $z=0$ is $\alpha F^{+}+(1-\alpha) F^{-}$, where

$$
\alpha F_{3}^{+}+(1-\alpha) F_{3}^{-}=0 \text {. }
$$

This implies that on the switching surface satisfying (3.4), the sliding flow is

$$
\dot{x}=\frac{F_{3}^{-} F_{1}^{+}-F_{3}^{+} F_{1}^{-}}{F_{3}^{-}-F_{3}^{+}}, \quad \dot{y}=\frac{F_{3}^{-} F_{2}^{+}-F_{3}^{+} F_{2}^{-}}{F_{3}^{-}-F_{3}^{+}} .
$$

It will be convenient to rescale the spatial variables by a factor of $|v|$ to emphasize the robust properties of the equations. In the rescaled variables, and ignoring higher order terms,

$$
F^{+}(\mathbf{x}, v)=\left(\begin{array}{ccc}
-\rho & a & -\omega \\
0 & \lambda & 0 \\
\omega & b & -\rho
\end{array}\right)\left(\begin{array}{c}
x \\
y \\
z-\operatorname{sgn}(v)
\end{array}\right)
$$

and

$$
F^{-}(\mathbf{x}, v)=\frac{1}{|v|}\left(\begin{array}{c}
U_{1} \\
U_{2} \\
U_{3}
\end{array}\right)
$$

The modified sliding evolution equations (3.10) are thus

$$
\dot{x}=\frac{U_{3} F_{1}^{+}-F_{3}^{+} U_{1}}{U_{3}-|v| F_{3}^{+}}, \quad \dot{y}=\frac{U_{3} F_{2}^{+}-F_{3}^{+} U_{2}}{U_{3}-|v| F_{3}^{+}},
$$

and by assumption the denominator is positive on the sliding surface for sufficiently small 
$|v|$ and so we may change the scaling of time whilst preserving the integral curves of solutions to consider

$$
\dot{x}=F_{1}^{+} U_{3}-F_{3}^{+} U_{1}, \quad \dot{y}=F_{2}^{+} U_{3}-F_{3}^{+} U_{2} .
$$

Thus, a sliding homoclinic orbit exists if there are values of the parameters such that (3.14) has a solution connecting $W_{u}$ defined by (3.8) to $W_{p}$ defined by (3.9) entirely in the sliding region (3.4).

For the choice of (3.1) above, the rescaled sliding equations (3.14) are

$$
\begin{aligned}
& \dot{x}=-\left(\rho U_{3}+\omega U_{1}\right) x+\left(a U_{3}-b U_{1}\right) y+\left(\omega U_{3}-\rho U_{1}\right), \\
& \dot{y}=-\omega U_{2} x+\left(\lambda U_{3}-b U_{2}\right) y-\rho U_{2} .
\end{aligned}
$$

To simplify the search for sliding homoclinic orbits, we will fix all the parameters except $\omega$, which will play the role of the Shilnikov parameter $\mu$ of Section 2.1, except that the bifurcation is shifted and will not be at $\omega=0$. We choose (with $v=1$ by the rescaling above)

$$
\rho=0.2, \quad a=4, \quad b=0.1, \quad U_{1}=-0.5, \quad U_{3}=1,
$$

for reasons described below. Two further conditions are imposed: $\lambda U_{3}=b U_{2}$ so that the coefficient in $y$ of the $\dot{y}$ equation of (3.15) vanishes making the calculation of pseudostationary points trivial; and $\rho / \lambda=\frac{3}{4}$ so that the Shilnikov condition $\rho<\lambda$ holds. These imply that

$$
\lambda=\frac{4}{3} \rho, \quad U_{2}=\frac{4 \rho U_{3}}{3 b}>0 .
$$

With these two conditions, the parameter values (3.16) are chosen so that the sliding flow (3.15) has a pseudostationary point which is an unstable focus if $\omega$ is order one - this seems sensible as the spiralling nature of this flow makes it quite sensitive to changes in initial conditions, making the search for sliding homoclinic orbits more likely to be successful, an intuition borne out by numerical experiments.

Finding a sliding homoclinic orbit numerically is now just a question of shooting using (3.15): If a sliding solution with initial condition $W_{u}$ first strikes the boundary of the sliding region with $y=0$, then the unstable manifold of the stationary point in $z>0$ is connected to the stable manifold of the stationary point by a sliding segment. Provided the orbit on the stable manifold does not strike the switching surface as it tends to the stationary point this will be a solution to the full equations. Numerical simulations suggests that there exist $\omega_{c} \approx 0.8846$ such that for $\omega$ a little below $\omega_{c}$ the solution on the sliding surface with initial condition $W_{u}$ defined by (3.8) first strikes $W_{b}$ in $y>0$, whilst if $\omega$ is a bit above $\omega_{c}$, then the first intersection is in $y<0$. Hence (up to numerical accuracy), there is a sliding homoclinic orbit; an approximation is shown in Figure 2.

We now need to discuss the implications of the existence of the sliding homoclinic orbit in this approximate system for the existence of such solutions in the original system (3.1), (3.2). First note that the existence of a (non-degenerate) connection between $W_{u}$ and $W_{p}$ at some $\omega_{c}$ for (3.15) with parameters given by (3.16) and (3.17) is stable to small perturbations of those constants, i.e. there will be values $\omega_{c}$ as a function of those parameters locally at which (3.15) has a solution from $W_{u}$ to $W_{p}$. 


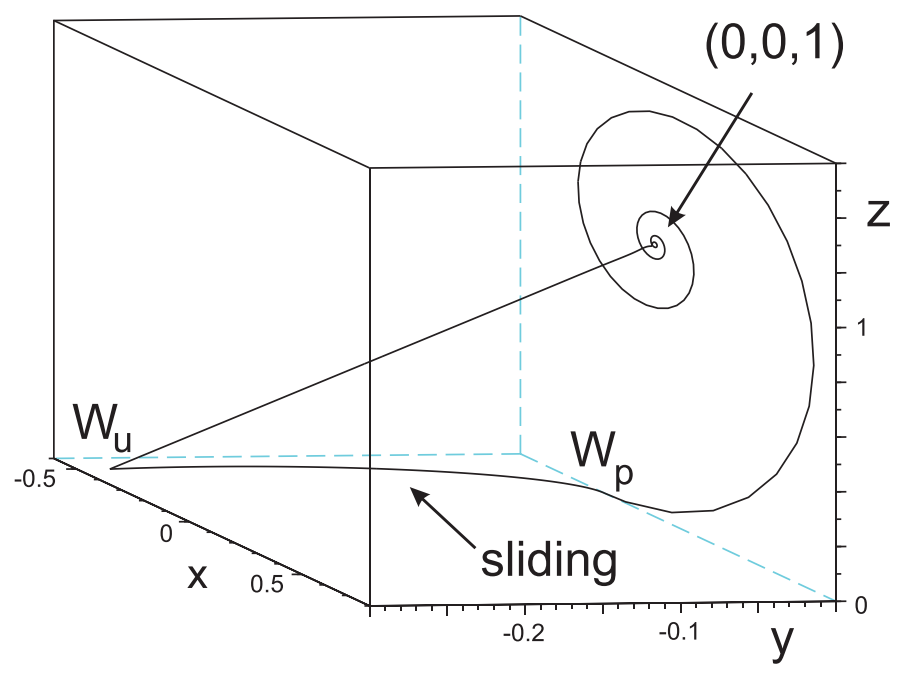

FIGURE 2. An approximate sliding homoclinic orbit of (3.11), (3.12) with parameters given by (3.16) and (3.17) and computed with $\omega=0.88504$ (see comments at the beginning of Section 4 about accuracy near stationary points).

Now fix the other parameters in this local neighbourhood so that $\omega_{c}$ exists. The approximations used to obtain (3.15) in the rescaled variables as $v \rightarrow 0$ were (i) ignore the higher order terms in (3.1) and (3.2), and then (ii) ignore the $|v| F^{+}$terms in the denominators of (3.13). If the terms of (ii) had been added in then generically, there is a curve $\omega_{h}(v)$ near $\mu=0$ with $\omega_{h}(0)=\omega_{c}$ such that if $\omega=\omega_{h}(v)$ then there is a connection from $W_{u}$ to $W_{p}$ of (3.13) and hence a sliding homoclinic orbit. Since the points $W_{u}$ and $W_{p}$ are relevant only if $v>0$, there is (generically) a half-line segment of $\omega_{h}(v)$ in $v>0$ locally. The original system is itself a small perturbation of the linearized system considered, and so for sufficiently small $v$, there will be sliding homoclinic bifurcations for the original system at values close to those of $\omega_{h}(v)$. In other words, there will be an open set of values of $\omega$ near $\omega_{c}$ (but not necessarily including $\omega_{c}$ ) which have sliding homoclinic orbits for small values of $v>0$. This justifies the 'open set' part of the conjecture in Section 2.3.

This does not complete the description of the BEB, which requires a description of the dynamics in $v<0$. Clearly, there will be many solutions near the switching surface that are unbounded, and although we have no rigorous proof, numerical simulations using a range of initial conditions suggest that almost all solutions do eventually leave a neighbourhood of the bifurcation. Thus, we conjecture that as a BEB, the transition is from systems with no local attractors to a systems with chaotic attractors.

\section{Further numerical simulations}

In this section, numerical methods are used to show the existence of double-pulse homoclinic orbits in the example of the previous section and verify the scaling results of (2.2) for the sliding homoclinic orbits. Before doing this, we give a brief discussion of the numerical methods used and the accuracy of results. 


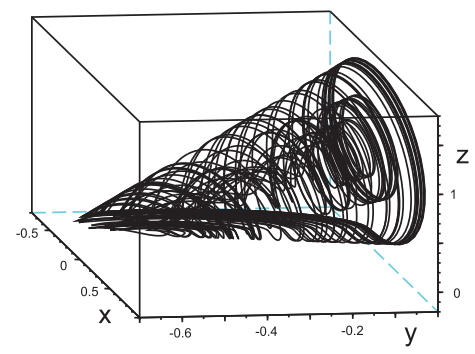

(a)

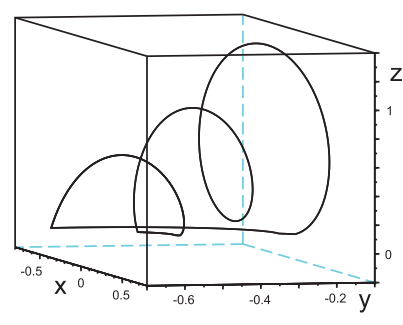

(b)

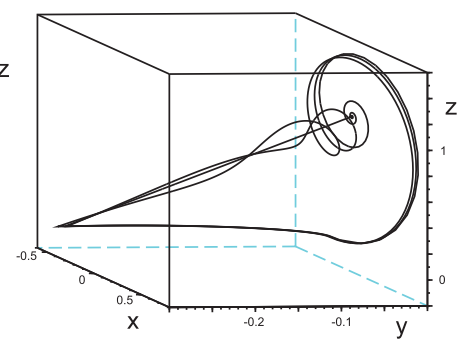

(c)

FIGURE 3. Orbits of (3.11), (3.12) with parameters given by (3.16) and (3.17) showing (a) a numerical chaotic attractor with $\omega=2.2$ and initial conditions $(-0.4549968,-0.5942628,0)$ on the sliding surface, (b) periodic orbit with two independent sliding segments with $\omega=2.93$ and initial conditions $(-0.1942009,-0.5086099,0)$ on the sliding surface and (c) approximate triple pulse homoclinic orbit computed with $\omega=0.893043381$.

Programmes were written in Scilab [27] using an ODE solver with a root finder. Generic methods to integrate Filippov systems do exist, e.g. [10,26], designed to avoid drift away from the sliding surface, but the equations here are sufficiently simple that the sliding equations can be written down and integrated explicitly, avoiding the problems the more sophisticated methods are designed to overcome. As well as the shooting method described in Section 3, homoclinic orbits can be found approximately by integrating solutions starting from $W_{u}$ through a neighbourhood of the stationary point once more to see whether they eventually exit into $y>0$ or $y<0$. Bisection again provides an approximate value of the homoclinic orbit, though if a long time is spent close to the stationary point, there may be no decision without spending longer integrating at a place where integration is least accurate. Whilst this is less accurate, it provides much more convincing pictures: all approximate homoclinic orbits depicted here are made up of trajectories starting at $W_{u}$ and integrated through the neighbourhood of the stationary point one extra time, exiting a neighbourhood of the stationary point very close to the local unstable manifold of the stationary point to provide the (approximate) part of the trajectory from the stationary point to $W_{u}$. This also makes it possible to check the global condition that there are no other intersections with the sliding surface. All the numerical experiments use the $v \rightarrow 0$ limit of the sliding equations (3.15).

It is easy to find (numerically) chaotic attractors for values of $\omega$ above the homoclinic bifurcation value $\omega_{c}$. One such is shown in Figure 3(a), and it would be tempting to say that this is explained by the Shilnikov theory of Section 2.3. However, a closer inspection shows that this cannot strictly be considered as local to the sliding homoclinic orbit: Solutions have sliding segments across broad range of the sliding surface, and at least some of this could be due to the existence of extra grazing bifurcations, see the stable periodic orbit of Figure 3(b).

Thus, in order to verify at least some of the theoretical predictions, we shall concentrate on the double-pulse solutions and their scaling (2.2). Figure 4 shows an (approximate) double-pulse homoclinic orbits found numerically. In fact eight were found at the values of $\omega$ shown in Table 1, where values are given found by the second method described above. We do not claim that the values are accurate to the given precision, but that the 


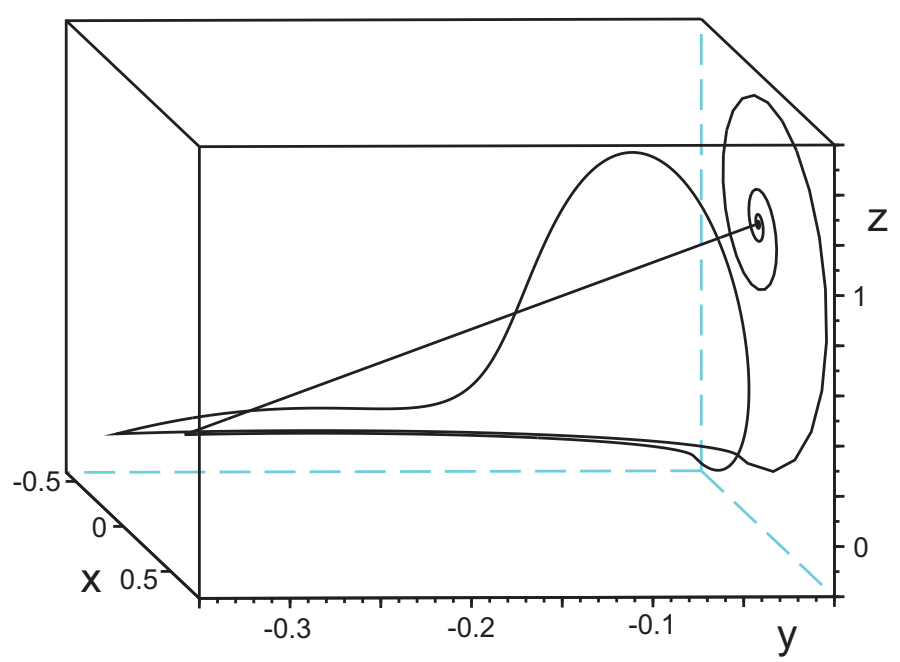

FIGURE 4. Approximate double-pulse sliding homoclinic orbits of (3.11), (3.12) with parameters given by (3.16) and (3.17) computed with $\omega=0.95493153$.

Table 1. Double pulse homoclinic orbits

\begin{tabular}{lll}
\hline \hline$n$ & \multicolumn{1}{c}{$\omega_{n}$} & $\delta_{n}$ \\
\hline 1 & 1.0435406 & \\
2 & 0.95493152 & 0.551 \\
3 & 0.90606445 & 0.268 \\
4 & 0.892948613 & 0.374 \\
5 & 0.888048141 & 0.383 \\
6 & 0.886170493 & 0.365 \\
7 & 0.8854839110 & 0.403 \\
8 & 0.885206889 & \\
\hline \hline
\end{tabular}

systematic errors are such that the differences used to calculate $\delta_{n}$ are useful. There is a further double-pulse homoclinic orbit at $\omega \approx 1.886$, but this seems to be beyond the range of the asymptotic theory so it has been ignored. This table also shows the six values of the right-hand side of (2.2),

$$
\delta_{n}=\frac{\omega_{n}-\omega_{n+1}}{\omega_{n-1}-\omega_{n}}
$$

which should be compared with the theoretical value

$$
\delta_{n} \rightarrow \exp \left(-\frac{\pi \lambda}{\omega}\right) \approx 0.375
$$

(where the right-hand side is evaluated at $\omega_{c}=0.885$ ). The agreement is good, though not spectacular, but since these are only the first few of an infinite sequence which become harder and harder to compute accurately, it seems a reasonable verification of the predicted asymptotic rate. 


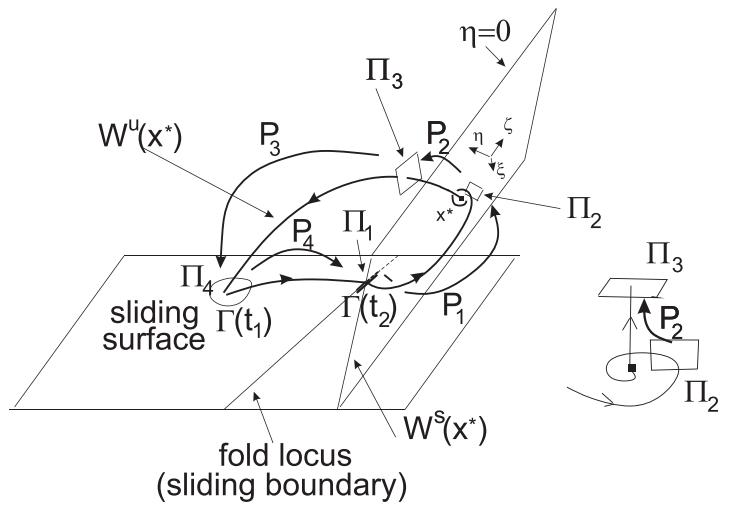

FiguRE 5. Sketch of the homoclinic orbit $\Gamma(t)$ with sliding section and the return planes on which the return maps are $P_{1}, \ldots, P_{4}$ are defined, with a blow up of the region near the stationary point. $\Gamma\left(t_{2}\right)$ is on the intersection of the stable manifold of the stationary point $x^{*}$ and the fold locus, so it corresponds to the point $W_{p}$ in Figure 2.

It is possible to find higher order $n$-pulse sliding homoclinic orbits: An example with $n=3$ is shown in Figure 3(c).

\section{Return maps near sliding homoclinic orbits}

In this section and the following Section 6, we prove Theorem 2.3 for general systems with sliding homoclinic orbits. Note that the map derived below is valid if $\rho$ and $\lambda$ are positive (i.e. for any saddle-focus) but the dynamics is very simple if $\rho>\lambda$ and so we restrict attention in Section 6 to the case $\rho<\lambda$ which generates the Shilnikov chaos in the smooth case.

With the notation of Section 2.3, solutions close to the sliding homoclinic orbit $\Gamma(t)$ can be divided into four segments as shown in Figure 5. A solution starting close to $\Gamma\left(t_{2}\right)$ on $\Pi_{1}$ in the fold locus (a one-dimensional surface) will map to a one-dimensional set in a two-dimensional surface $\Pi_{2}$ close to $\mathbf{x}^{*}$. Call this map $P_{1}$. Solutions on $\Pi_{2}$ close to $\mathbf{x}^{*}$ pass through a neighbourhood of $\mathbf{x}^{*}$ where the evolution is dominated by the linear part of the differential equation and leave this neighbourhood on a two-dimensional surface $\Pi_{3}$ provide they leave a neighbourhood of $x^{*}$ close to the correct branch of its local unstable manifold - the branch close to $\Gamma(t)$ with $t$ large and negative. Call this return map $P_{2}$. Solutions on $\Pi_{3}$ will strike $s(\mathbf{x}, \mu)=0$ close to $\Gamma\left(t_{1}\right)$ in $\Pi_{4}$. Call this map $P_{3}$ and note that condition (iv) of Section 2.3 implies that we may choose $\Pi_{4}$ so that it does not contain any fold loci. Finally, solutions on $\Pi_{4}$ will be mapped close to $\Gamma\left(t_{2}\right)$ on the fold locus by a map $P_{4}$ which takes the two-dimensional area $\Pi_{4}$ to a one-dimensional segment containing $\Pi_{1}$. The maps $P_{2}$ and $P_{3}$ are effectively standard. The new ingredients here are the maps $P_{1}$ and $P_{4}$. These four maps will then be composed to give a map from the fold locus to itself describing nearby solutions as they pass through a neighbourhood of $\Gamma(t)$.

Technical Remark: Error terms depend on the degree of smoothness of the defining vector fields required to change coordinates so that in a neighbourhood of a stationary 
point the flow is linear in the new coordinate system. For the $C^{1,1}$ case adopted in Section 1, Tresser [32,33] state results as being $C^{1}$ close to the leading-order terms, whilst with analytic vector fields, it is possible to give the order of the errors in terms of the "big O' notation [13], although there are complications in resonant cases. The choice of $C^{3}$ vector fields in Section 1 makes it possible to use linearisation results of Sell [28]. If the eigenvalues $\left(\lambda_{k}\right)$ of the Jacobian matrix at the stationary point satisfy a relationship of the form $\lambda_{i}=\sum m_{k} \lambda_{k}$ for non-negative integers $m_{k}$ and $|m|=\sum m_{k}$, then the stationary point is said to be resonant of order $|m|$. Sell [28] shows that a $C^{3}$ change of coordinates can be used to linearize a $C^{3}$ vector field near a stationary point if it is non-resonant or resonant of order greater than three. In our case, with eigenvalues $\lambda$ and $-\rho \pm i \omega$, a relationship of the form $\lambda=m_{1} \lambda+m_{2}(-\rho+i \omega)+m_{3}(-\rho-i \omega)$ requires $m_{2}=m_{3} \geqslant 1$ and $m_{1} \geqslant 2$ on grounds of signs alone. Hence, $|m| \geqslant 4$ and so Shilnikov saddle-foci in $\mathbb{R}^{3}$ can be linearized with $C^{3}$ transformations. We are not aware of Sell's results being used in this context before, but they appear to be a very natural extension of the linearization theorems $[3,4,31]$ that are usually quoted. For reasons of space, only the leading-order terms will be given in the discussion below, but since transformations are $C^{3}$, the quadratic error terms of e.g. [13] hold here.

Since the form of the map $P_{1}$ depends on the local coordinate system defined in a neighbourhood of $\mathbf{x}^{*}(\mu)$ which is chosen in the construction of $P_{2}$, it is natural to begin the description of these maps with $P_{2}$.

\subsection{The map $P_{2}[13,16,30]$}

We may choose coordinates centred on $\mathbf{x}^{*}(\mu)$ such that the differential equation locally is linear (or locally linear to lowest order in [13]). In these coordinates $(\xi, \zeta, \eta)$, the equation is

$$
\dot{\xi}=-\rho \xi-\omega \zeta, \quad \dot{\zeta}=\omega \xi-\rho \zeta, \quad \dot{\eta}=\lambda \eta .
$$

$\Gamma(t)$ intersects the plane $\xi=0$ infinitely often as it spirals into the origin with $\eta=0$. Choose one such intersection point, $(0, R, 0)$ with $0<R \ll 1$ and let $\Pi_{2}$ be the intersection of a neighbourhood of $(0, R, 0)$ with

$$
\{(\xi, \zeta, \eta)|\xi=0 .| \zeta-R|\ll 1,| \eta \mid \ll 1\}
$$

chosen so that solutions with initial conditions on $\Pi_{2}$ intersect $\Pi_{2}$ once as they spiral towards the fixed point and away in the $\eta$ direction. Without loss of generality, assume that the branch of the unstable manifold of the origin which creates the homoclinic orbit if $\mu=0$ lies in $\eta>0$. If $\eta<0$, then solutions with initial conditions on $\Pi_{2}$ leave $-R<\eta<R$ on $\eta=-R$ and are lost to the analysis. Let

$$
\Pi_{3}=\{(\xi, \zeta, \eta)|\eta=R,| \xi|\ll 1,| \zeta \mid \ll 1\} .
$$

If $\eta>0$, then solutions strike $\Pi_{3}$ close to $(0,0, R)$ after time $\tau$, where

$$
R=\eta e^{\lambda \tau} \quad \text { or } \quad e^{-\tau}=\left(\frac{\eta}{R}\right)^{\frac{1}{\lambda}}
$$


Thus, for initial conditions on $\Pi_{2}, \mathbf{x}=\mathbf{x}^{*}(\mu)+(0, \zeta, 0, \eta)$, the solution at time $\tau$ in polar coordinates $(r, \theta)$ in the $(\xi, \zeta)$ plane is

$$
r=\zeta e^{-\rho \tau}, \quad \theta=\omega \tau
$$

and hence, since $\zeta \sim R$ to lowest order, if $\delta=\rho / \lambda$, the intersection of the solution on $\Pi_{3}$ is approximately

$$
\xi \sim R\left(\frac{\eta}{R}\right)^{\delta} \cos \left(-\frac{\omega}{\lambda} \ln \eta+\Phi\right), \quad \zeta \sim R\left(\frac{\eta}{R}\right)^{\delta} \sin \left(-\frac{\omega}{\lambda} \ln \eta+\Phi\right), \quad \eta>0,
$$

for an appropriate choice of the phase shift $\Phi$. We have not gone through the full detail of this argument here, see e.g. [13] for details of the size of the error terms.

\subsection{The map $P_{3}$}

From $(0,0, R)$ on $\Pi_{3}$ in $(\xi, \zeta, \eta)$ coordinates, the unstable manifold strikes $s(\mathbf{x}, \mu)$ at a point order $\mu$ away from $\Gamma\left(t_{1}\right)$. Assuming that the tangent plane to $s$ is well-defined here, and since $\Gamma(t)$ is transverse to this plane at the point of intersection by assumption, coordinates $(X, Y, Z)$ can be chosen near $\Gamma\left(t_{1}\right)$ so that $s=0$ is $Z=0$, which we call $\Pi_{4}$ and $\Gamma\left(t_{1}\right)=(0,0,0)$ in these coordinates. The flow from $\Pi_{3}$ to $\Pi_{4}$ is $C^{3}$ and the coordinate change is also $C^{3}$, so we may expand the return map $P_{3}: \Pi_{3} \rightarrow \Pi_{4}$ locally as

$$
P_{3}(\xi, \zeta) \sim\left(\begin{array}{l}
X \\
Y
\end{array}\right)=\left(\begin{array}{c}
M \\
N
\end{array}\right) \mu+\left(\begin{array}{ll}
A & B \\
C & D
\end{array}\right)\left(\begin{array}{l}
\xi \\
\zeta
\end{array}\right)
$$

where $A, B, C, D, M, N$ are constants.

\subsection{The map $P_{1}$}

If $\mu=0$, the homoclinic orbit takes the point $\Gamma\left(t_{2}\right)$ to $(R, 0,0)$ in coordinates $(\xi, \zeta, \eta)$ on $\Pi_{2}$. Nearby points on the fold locus $\Pi_{1}$ are mapped close to this, the flow is transverse to the fold locus as it is tangential to $s=0$, and solutions are not close to singularities of the flow so this is again a $C^{3}$ function of $C^{3}$ coordinates, and choosing a coordinate $w$ on $\Pi_{1}$ such that $w=0$ corresponds to $\Gamma\left(t_{2}\right)$, we find $P_{1}: \Pi_{1} \rightarrow \Pi_{2}$ is

$$
P_{1}(w) \sim\left(\begin{array}{c}
\zeta^{\prime} \\
\eta^{\prime}
\end{array}\right)=\left(\begin{array}{c}
M_{1} \\
N_{1}
\end{array}\right) \mu+\left(\begin{array}{c}
A_{1} \\
B_{1}
\end{array}\right) w
$$

to lowest order, where $A_{1}, B_{1}, M_{1}, N_{1}$ are constants.

\subsection{The map $P_{4}$}

The map $P_{4}: \Pi_{4} \rightarrow \Pi_{1}$ is the least standard of the return maps, though really it is just a variant of $P_{1}$. Close to $\Gamma\left(t_{1}\right)$ on $\Pi_{4}$ coordinates $(X, Y)$ can be chosen so that the flow is parallel to the $X$-axis. In these new coordinates, the coefficients of (5.4) are changed but not the form. Assume this has been done. Then all initial conditions on $\Pi_{4}$ with the same $Y$ coordinate map to the same point on $P_{1}$, i.e. it is independent of the $X$ coordinate. The 
flow is trajectories are those of a $C^{3}$ system locally (as the solution from $\Gamma\left(t_{1}\right)$ to $\Gamma\left(t_{2}\right)$ is bounded away from any pseudostationary points), and hence to lowest order

$$
P_{4}(X, Y) \sim w^{\prime}=\left(\begin{array}{c}
M_{4} \\
N_{4}
\end{array}\right) \mu+\left(\begin{array}{c}
A_{4} \\
B_{4}
\end{array}\right) Y,
$$

where $A_{4}, B_{4}, M_{4}, N_{4}$ are constants. Note that this map is not invertible reflecting the fact that initial conditions on $\Pi_{4}$ can lie on the same sliding trajectory.

\section{The composed map: chaos and periodic orbits}

The map $P: \Pi_{1} \rightarrow \Pi_{1}$ with $P=P_{4} \circ P_{3} \circ P_{2} \circ P_{1}$ is the full return map on the onedimensional fold locus near $\Gamma\left(t_{2}\right)$. This is not defined if $N_{1} \mu+B_{1} w \leqslant 0$ as this leads to intersections with $\Pi_{2}$ having $\eta<0$ and these are on the wrong side of the stable manifold of the stationary point and hence are lost from the analysis (or tend to the stationary point in the case of equality). All other solutions can be followed back to $\Pi_{1}$, although of course they may return in the forbidden zone and be lost at the next iteration. Composing the map as defined, it is clear that $w_{n+1}=P\left(w_{n}\right)$ will be a linear combination of the terms in (5.3) plus a multiple of $\mu$ to lowest order, thus we may write

$$
w_{n+1}=P\left(w_{n}\right) \sim a_{1} \mu+b_{1}\left(N_{1} \mu+B_{1} w_{n}\right)^{\delta} \cos \left(\Xi \log \left(N_{1} \mu+B_{1} w_{n}\right)+\Psi\right),
$$

for constants $a_{1}, b_{1}$ and $\Psi$ which are functions of the constants defining the linear part of the maps, and $\Xi=-\frac{\omega}{\lambda}$, valid if $N_{1} \mu+B_{1} w>0$. Let $v_{n}=N_{1} \mu+B_{1} w_{n}$, then

$$
v_{n+1}=P_{*}\left(v_{n}\right) \sim a \mu+b v_{n}^{\delta} \cos \left(\Xi \log v_{n}+\Psi\right), \quad v_{n}>0,
$$

with $b=b_{1} B_{1}$ and $a=N_{1}+a_{1} B_{1}$, and it is this map that we will describe in more detail under the generic assumption that $a$ and $b$ are non-zero. This is a non-invertible one-dimensional map with infinitely many turning points. The equation for fixed points, $v_{n+1}=v_{n}$, is the lowest order approximation to the fixed point equation of the twodimensional map derived in [16]. Glendinning and Sparrow [16] are careful to emphasize that this is not a one-dimensional map in the classic Shilnikov case. In contrast, here it is really the one-dimensional lowest order model of the flow.

The analysis of (6.2) now follows the analysis of [16] and [13] for the smooth case, so only a sketch of the arguments will be given.

Fixed points correspond to orbits that pass once through a neighbourhood of $\Gamma(t)$ on each period. Since $\delta<1$ and by assumption $v$ is small, $v^{\delta} \gg v$ and so the locus of fixed points in the $(v, \mu)$ plane is (to lowest order)

$$
\mu \sim-\frac{b}{a} v^{\delta} \cos (\Xi \log v+\Psi) .
$$

Clearly, there are countably many fixed points if $\mu=0$ and a finite number if $\mu \neq 0$ [16]. The coordinate $v$ is essentially a rescaling of $\eta$ on $\Pi_{2}$ and hence (5.2) implies that $\ln v \sim-\lambda \tau$ where $\tau$ is the time spent in a small neighbourhood of the stationary point $\mathbf{x}^{*}$, and hence as $v \rightarrow 0$ and $\mu \rightarrow 0$ the period of the orbit, $T$, is approximately $\tau$ and the 
fixed point equation (6.3) can be written as a curve in the $(\mu, T)$-space as

$$
\mu \sim-\frac{b}{a} e^{-\rho T} \cos (-\omega T+\Psi)
$$

as $T \rightarrow \infty$. In this limit, $\mu \rightarrow 0$, i.e. the orbit converges on the homoclinic orbit $\Gamma(t)$.

The fixed points are created or destroyed in saddle node bifurcations when the derivative of the right-hand side of (6.2) equals to +1 , and since that derivative is proportional to $v^{\delta-1} \gg 1$, then to lowest order saddle node bifurcations occur at (i.e. very close to) the turning points of the map. This implies that they occur at parameters $\mu_{n} \rightarrow 0$ with

$$
\frac{\mu_{n+1}}{\mu_{n}} \sim-\left(\frac{v_{n+1}^{t}}{v_{n}^{t}}\right)^{\delta} \sim-\exp \left(-\frac{\pi \rho}{\omega}\right)
$$

showing that the saddle node bifurcations oscillate on either side of $\mu=0$ and rearranging a little gives the expression in Theorem 2.3.

Further results on these orbits can be derived from (6.2). For example, the condition $\delta<1$ implies that there are complete sequences of bifurcations and chaos as in the logistic map and their inverse cascades associated with each pair of stable fixed points created in the saddle node bifurcations.

If $\mu=0$, then there is a countable (infinite) number of horseshoes in the dynamics: each of the 'humps' of the return map is chaotic. For sufficiently small $|\mu| \neq 0$, a finite number of these persist. If $\mu=0$, the maxima and minima of the return map (6.2) occur approximately at $v_{n}$ defined above, and the maximum will therefore be approximately $b v_{n}^{\delta}$. Thus, there will be two branches of the map near $v_{n}$ that cover $v_{k}$ for all $k$ such that $v_{k}<b v_{n}^{\delta}$. Writing $v_{n} \sim \alpha e^{-\pi \lambda n / \omega}$, so $v_{n}^{\delta} \sim \alpha^{\delta} e^{-\pi \rho n / \omega}$, this becomes

$$
\alpha e^{-\pi \lambda k / \omega}<b \alpha^{\delta} e^{-\pi \rho n / \omega}
$$

i.e. for all $k$ such that

$$
k>C+\delta n
$$

where $C$ is a constant. This can be expressed as $k / n>\delta+C / n$ and so as $n \rightarrow \infty, k / n>\delta$. In other words, the chaotic set contains a set that is conjugate to of all sequences of symbols $\{K, K+1, K+2, \ldots\}^{\mathbb{N}}$ ( $K$ sufficiently large) such that if the sequence is $\left(s_{0}, s_{1}, \ldots\right)$, then $s_{n+1}>\delta s_{n}$. This is non-trivial since the condition $\rho<\lambda$ implies that $\delta>1$ [30].

More complicated homoclinic orbits exist near $\mu=0$ for the smooth version of Shilnikov chaos. These are also present in the sliding case. The simplest example is a homoclinic orbit with two loops close to $\Gamma(t)$, these are called double-pulse homoclinic orbits.

The unstable manifold of the stationary point intersects $\Pi_{3}$ at $(\xi, \zeta)=(0,0)$ It therefore maps to $\Pi_{1}$ at $P_{4} \circ P_{3}(0,0) \sim M_{5} \mu$, where $M_{5}$ is a constant obtained from (5.4) and (5.6) whose values do not matter greatly. There will be a double-pulse homoclinic orbit if $N_{1} \mu+B_{1} M_{5} \mu>0$ so that the map (6.2) can be applied, and then that at the next iteration the image is $v=0$ (which means that at the next iteration the orbit lands on the stable manifold of the stationary point). Thus, if $\beta=N_{1}+B_{1} M_{5} \neq 0$, the condition for the existence of a double-pulse homoclinic orbit is that

$$
\beta \mu>0, \quad a \mu+b(\beta \mu)^{\delta} \cos (\Xi \log (\beta \mu)+\Psi)=0,
$$


to lowest order. The first of these conditions is satisfied provided $\mu$ takes the appropriate sign (the sign of $\beta$ ) and given that, there will be an infinite sequence $\tilde{\mu}_{n} \rightarrow 0$ of solutions to the second equation with

$$
\Xi \log \left|\tilde{\mu}_{n+1}\right| \sim \Xi \log \left|\tilde{\mu}_{n}\right|+\pi
$$

or

$$
\frac{\tilde{\mu}_{n+1}}{\tilde{\mu}_{n}} \sim \exp \left(-\frac{\pi \lambda}{\omega}\right)
$$

leading to the convergence result expressed in the statement of Theorem 2.3.

More complicated $n$-pulse homoclinic orbits exist and can be described using the methods of [11].

\section{Conclusion}

We have shown how the homoclinic bifurcation theory of Shilnikov [30] applies to homoclinic orbits of piecewise smooth systems with a sliding segment. Analysis of a three-dimensional BEB shows that this is a mechanism associated with the creation of complex orbits near other bifurcations of piecewise smooth systems. Finally, we have seen how further complications can be introduced due to the geometry of the switching manifold and the homoclinic orbit, making grazing bifurcations possible. All of these have been illustrated by simple examples.

The work of Filippov [12] is almost entirely planar, as is much of the more recent analysis. This paper begins an exploration of bifurcation mechanisms in higher dimensional piecewise smooth dynamical systems, and whilst it answers some questions, it provides evidence for more complicated interactions of bifurcations that merit further analysis.

\section{References}

[1] Arnéodo, A., Coullet, P. \& Tresser, C. (1981) Possible new strange attractors with spiral structure. Commun. Math. Phys. 79(4), 573-579.

[2] Arnéodo, A., Coullet, P. \& Tresser, C. (1982) Oscillators with chaotic behavior: An illustration of a theorem by Shil'nikov. J. Stat. Phys. 27(1), 171-182.

[3] Belitskil, G. R. (1973) Functional equations and conjugacy of local diffeomorphisms of a finite smoothness class. Funct. Anal. Appl. 7(4), 268-277.

[4] Chen, K. T. (1963) Equivalence and decomposition of vector fields about an elementary critical point. Amer. J. Math. 85(4), 693-722.

[5] Colombo, A. \& Jefreey, M. R. (2013) The two-fold singularity: Leading order dynamics in n-dimensions. Physica D 263, 1-10.

[6] di Bernardo, M., Budd, C., Champneys, A. R. \& KowalczyK, P. (2008) Piecewise-Smooth Dynamical Systems, Springer, London.

[7] di Bernardo, M., Pagano, D. J. \& Ponce, E. (2008) Nonhyperbolic boundary equilibrium bifurcations in planar Filippov systems: A case study approach. Int. J. Bifurcation Chaos 18(5), 1377, DOI: $10.1142 / \mathrm{S} 0218127408021051$.

[8] de Carvalho, T. \& Tonon, D.J. (2014) Normal forms for codimension one planar piecewise smooth vector fields. Int. J. Bifurcation Chaos 24(7), 1450090, DOI: $10.1142 / \mathrm{S} 0218127414500904$ 
[9] Dercole, F., Della Rossa, F., Colombo, A. \& Kuznetsov, Y. A. (2011) Two degenerate boundary equilibrium bifurcations in planar Filippov systems. SIADS 10(4), 1525-1553.

[10] Dieci, L. \& Lopez, L. (2009) Sliding motion in Filippov differential equations: Theoretical results and a computational approach. SIAM J. Numer. Anal. 47(3), 2023-2051.

[11] Feroe, J. A. (1993) Homoclinic orbits in a parametrized saddle-focus system. Physica D 62(1-4), 254-262.

[12] Filippov, A. F. (1988) Differential Equations With Discontinuous Right Hand Sides, Kluwer, Netherlands.

[13] Gaspard, P., Kapral, R. \& Nicolis, G. (1984) Bifurcation phenomena near homoclinic systems: A two-parameter analysis. J. Stat. Phys. 35(5-6), 697-727.

[14] Glendinning, P. (1997) Differential equations with bifocal homoclinic orbits. Int. J. Bifurcation Chaos 7(1), 27-37.

[15] Glendinning, P. (2016) Classification of boundary equilibrium bifurcations of planar Filippov systems. Chaos 26, 013108.

[16] Glendinning, P. \& Sparrow, C. (1984) Local and global behavior near homoclinic orbits. J. Stat. Phys. 35(5-6), 645-696.

[17] Guardia, M., Seara, T. M. \& Teixeira, M. A. (2011) Generic bifurcations of low codimension of planar Filippov Systems. J. Diff. Equ. 250(4), 1967-2023.

[18] Hogan, S. J., Homer, M. E., Jeffrey, M. R. \& Szalai, R. (2016) Piecewise smooth dynamical systems theory: The case of the missing boundary equilibrium bifurcations. J. Nonl. Sci. 26(5), 1161-1173.

[19] Hös, C. \& ChampNeys, A. R. (2012) Grazing bifurcations and chatter in a pressure relief valve model. Physica D 241(22), 2068-2076.

[20] Jeffrey, M. R. \& Colombo, A. (2009) The two-fold singularity of discontinuous vector fields. SIADS 8(2), 624-640.

[21] Kuznetsov, Y. A., Rinaldi, S. \& Gragnani, A. (2003) One-parameter bifurcations in planar Filippov systems. Int. J. Bifurcation Chaos 13(8), 2157-2188.

[22] Llibre, J., Ponce, E. \& Teruel, A. E. (2007) Horseshoes near homoclinic orbits for piecewise linear differential systems in $\mathbb{R}^{3}$. Int. J. Bifurcation Chaos 17(4), 1171-1184.

[23] Matsumoto, T., Chua, L. O. \& Komuro, M. (1985) The double scroll. IEEE Trans. Circ. Syst. 32(8), 797-818.

[24] Novaes, D. D. \& Teixeira, M. A. (2015) Shilnikov problem in Filippov dynamical systems, arXiv.

[25] Nusse, H. E. \& Yorke, J. A. (1992) Border-collision bifurcation including 'period two to period three' for piecewise smooth systems. Physica D 57(1-2), 39-57.

[26] Pirroinen, P. \& Kuznetsov, Y. A. (2008) An event-driven method to simulate Filippov systems with accurate computing of sliding motions. ACM Trans. Math. Softw. 34(3), Art. 13.

[27] Orsay (2012) Scilab: Free and Open Source Software. URL: http: \\www.scilab.org (last accessed 4 June 2018).

[28] Sell, G. R. (1985) Smooth linearization near a fixed point. Amer. J. Math. 107(5), 1035-1091.

[29] Shilnikov, L. P. (1965) A case of the existence of a denumerable set of periodic motions. Sov. Math. Dokl. 6, 163-166.

[30] Shilnikov, L. P. (1970) A contribution to the problem of the structure of an extended neighborhood of a rough equilibrium state of saddle-focus type. Math. USSR Sb. 10(1), 91-102.

[31] Sternberg, S. (1959) The structure of local homoeomorphisms III. Amer. J. Math. 81(3), 578-604.

[32] Tresser, C. (1983) Un théorème de Sil'nikov en $C^{1,1}$. C.R.Acad. Sci. Série I 296, 545-548.

[33] Tresser, C. (1984) About some theorems by L.P. Sil'nikov. Ann. de l'IHP 40(4), 441-461. 


\section{Appendix A Normal form calculation}

We shall approach the normal form for a BEB of an equilibrium with Jacobian having eigenvalues $\lambda$ and $-\rho \pm i \omega$ from a geometric point of view. Suppose that the switching manifold is locally the $(x, y)$-plane, $z=0$, and that at the bifurcation value of parameters the equilibrium is at the origin. By definition, the equilibrium has a two-dimensional invariant subspace tangential to the local stable manifold, $E^{s}$, and generically this intersects the $(x, y)$-plane transversely on a line. Choose the $x$-direction, with unit vector $\mathbf{e}_{1}$ parallel to this direction and the $y$-direction, with unit vector $\mathbf{e}_{2}$ to be orthogonal to $\mathbf{e}_{1}$. Finally, let $\mathbf{e}_{3}$ be the unit vector in the positive $z$-direction.

The local stable manifold $E^{s}$ of the linearized flow is spanned by $\mathbf{e}_{1}$ and a second unit vector $\mathbf{e}_{s}$ perpendicular to $\mathbf{e}_{1}$, so we may choose

$$
\mathbf{e}_{s}=p \mathbf{e}_{2}+q \mathbf{e}_{3}, \quad q>0, \quad p^{2}+q^{2}=1 .
$$

If the linearized flow is

$$
\dot{\mathbf{x}}=L \mathbf{x}
$$

then the invariance of $E^{s}$ implies that

$$
L \mathbf{e}_{1}=\alpha \mathbf{e}_{1}+\beta \mathbf{e}_{s}, \quad L \mathbf{e}_{s}=\gamma \mathbf{e}_{1}+\delta \mathbf{e}_{s},
$$

and we may write

$$
L \mathbf{e}_{2}=u \mathbf{e}_{1}+v \mathbf{e}_{2}+w \mathbf{e}_{s},
$$

for constants $\alpha, \beta, \gamma, \delta, u, v$ and $w$.

With the obvious abuse and reuse of the symbol $z$, let

$$
\mathbf{x}=x \mathbf{e}_{1}+y \mathbf{e}_{2}+z \mathbf{e}_{s}
$$

so the sliding surface is the set $z=0$ in these coordinates too and

$$
\dot{\mathbf{x}}=\dot{x} \mathbf{e}_{1}+\dot{y} \mathbf{e}_{2}+\dot{z} \mathbf{e}_{s}
$$

Using (A 3) and (A 4),

$$
L \mathbf{x}=x\left(\alpha \mathbf{e}_{1}+\beta \mathbf{e}_{s}\right)+y\left(u \mathbf{e}_{1}+v \mathbf{e}_{2}+w \mathbf{e}_{s}\right)+z\left(\gamma \mathbf{e}_{1}+\delta \mathbf{e}_{s}\right),
$$

and by (A 2) the right-hand sides of (A 6) and (A 7) are equal.

Taking the scalar product of these right-hand sides with $\mathbf{e}_{1}, \mathbf{e}_{2}$ and $\mathbf{e}_{s}$, respectively, noting that $\mathbf{e}_{1} \cdot \mathbf{e}_{s}=p$ and $\mathbf{e}_{2} \cdot \mathbf{e}_{s}=q$ by (A 1) gives

$$
\begin{aligned}
\dot{x}+p \dot{z} & =(\alpha+p \beta) x+(\gamma+p \delta) z \\
\dot{y}+q \dot{z} & =q \beta x+(v+w q) y+q \delta z \\
p \dot{x}+q \dot{y}+\dot{z} & =(p \alpha+\beta) x+(p u+q v+w) y+(p \gamma+\delta) z
\end{aligned}
$$


Standard manipulations show that this set of equations simplifies to

$$
\begin{aligned}
& \dot{x}=\alpha x+s y+\gamma z, \\
& \dot{y}=v y, \\
& \dot{z}=\beta x+t y+\delta z,
\end{aligned}
$$

where $s$ and $t$ are constants. Hence, $v=\lambda$ and only the first and third equations of (A 9) need further consideration. This two-dimensional part of the argument is similar to that of [15].

Let

$$
X=x+A z
$$

then $\dot{X}=(\alpha+A \beta) x+(s+A t) y+(\gamma+A \delta) z$, so

$$
\begin{aligned}
\dot{X} & =(\alpha+A \beta) X+(s+A t) y+\left(\gamma+(\delta-\alpha) A-\beta A^{2}\right) z, \\
\dot{z} & =\beta x+t y+(\delta-A \beta) z .
\end{aligned}
$$

Provided $\beta \neq 0$, the choice $A=(\delta-\alpha) /(2 \beta)$ gives

$$
\begin{aligned}
\dot{X} & =-\rho X-+(s+A t) y+\gamma^{\prime} z, \\
\dot{z} & =\beta x+t y-\rho z,
\end{aligned}
$$

where $-\rho=\frac{1}{2}(\alpha+\delta)$ and $\gamma^{\prime}$ is constant. Since the eigenvalues of the Jacobian matrix of the two-dimensional system in $(X, z)$ evaluated at the origin are $-\rho \pm i \omega$, the product $\beta \gamma^{\prime}=-\omega^{2}$, so we may write $\gamma^{\prime}=-\kappa^{2} \omega$ and then $\beta=\kappa^{-2} \omega$. Now setting $Z=\kappa^{2} z$, the full equations become

$$
\begin{aligned}
\dot{X} & =-\rho x+a y-\omega z, \\
\dot{y} & =\lambda y, \\
\dot{z} & =\omega x+b y-\rho z .
\end{aligned}
$$

The linear part of this is now precisely the form of (3.1).

If (A 11) is the flow in the upper half-plane, then the unfolding of the BEB is obtained by adding a parameterized vector, which after a further shift of the origin if necessary can be taken to give a system with an equilibrium at $(0,0, v)$, where $v$ is the unfolding parameter, and non-linear terms in the parameter and the phase variables which are small compared with the constant and linear terms.

Provided they do not vanish locally, the transformations change the values of the constant linear flow in the lower half-plane, and the sign of the $Z$-component is unchanged by the transformations, hence the full normal form is as described in Section 3. 